05.3;06.5

\title{
Теплопроводность композита на основе н-алкана и наноразмерных добавок
}

\author{
(C) В.М. Егоров, А.К. Борисов, В.А. Марихин \\ Физико-технический институт им. А.Ф. Иофрфе РАН, Санкт-Петербург, Россия \\ E-mail: victor_egorov1@inbox.ru
}

Поступило в Редакцию 10 июня 2021 г.

В окончательной редакции 16 сентября 2021 г.

Принято к публикации 27 сентября 2021 г.

Для выяснения эффективности теплопреобразования при фазовых переходах в РСM (phase change materials) исследовались композиты на основе н-алкана и наноразмерных добавок. Обосновано применение нового метода измерения теплопроводности посредством анализа формы пиков, регистрируемых на термограмме методом дифференциальной сканирующей калориметрии. Установлено, что теплопроводность композитных материалов в несколько раз превышает аддитивную теплопроводность исходных компонентов. Обнаруженный эффект обусловлен спецификой надмолекулярной наноструктуры композита, отличной от надмолекулярной структуры компонентов.

Ключевые слова: теплопроводность, фазовый переход, композит, н-алканы.

DOI: 10.21883/PJTF.2022.02.51911.18915

Одним из наиболее актуальных и перспективных направлений развития зеленой энергетики является разработка PCM (phase change materials), способных поглощать, хранить и преобразовывать потоки тепловой энергии за счет собственных тепловых эффектов, в том числе при фазовых переходах (ФП). В качестве наиболее перспективных в этом отношении РСМ рассматриваются гомологические ряды алифатических углеводородов и их производных, в первую очередь нормальные н-алканы [1-3]. Помимо наиболее важной характеристики РСМ - их энергоемкости - важное значение имеет теплопроводность этих материалов, определяющая мощность поглощаемой или выделяемой в виде тепла энергии. Широкое распространение метода дифференциальной сканирующей калориметрии (ДСК), обусловленное его экспрессностью и информативностью, позволило разработать метод определения коэффициента теплопроводности для различных материалов: полимеров, металлов, керамик [4]. Этот метод основан на анализе формы пиков теплоемкости $C_{p}(T)$, вызванных фазовыми переходами (твердотельными переходами, плавлением), и сравнении этих пиков с пиками теплоемкости в эталонных материалах.

В настоящей работе для выяснения эффективности теплопреобразования при фазовых переходах исследовались композиты на основе н-алкана (нонадекана) и наноразмерных добавок: исходный нонадекан (образец I), нонадекан с добавлением частиц $\mathrm{Al}_{2} \mathrm{O}_{3}$ размером 50-70 nm (образец II), нонадекан с добавлением частиц $\mathrm{Al}_{2} \mathrm{O}_{3}$ размером 90-110 nm (образец III), нонадекан с добавлением частиц $\mathrm{Ag}$ размером 90-100 nm (образец IV). Образцы композитов готовились в весовой пропорции нонадекан/порошок 95/5. Для равномерного распределения добавок по объему образцы в расплавленном состоянии подвергались обработке на ультразвуковом диспергаторе УЗД1-0,1/22.

Термодинамические параметры композитов определялись на калориметре ДСК-500 фирмы „Спецприбор“ в атмосфере азота при скорости сканирования $1 \mathrm{~K} / \mathrm{min}$. Температурная шкала калибровалась по точкам плавления льда $(273.1 \mathrm{~K})$ и индия $(429.7 \mathrm{~K})$, шкала теплового потока - по теплоемкости лейкосапфира. Измерения проводились в области температур 270-440 К. Вес образцов для испытаний составлял $5 \mathrm{mg}$.

Как было отмечено выше, в работе [4] был разработан метод определения коэффициента теплопроводности путем анализа формы эндотермического пика фазового перехода. Показано, что наклон пика на экспериментальной кривой ДСК испытываемого образца и смещение температуры максимума пика $T_{\max }$ к более высоким температурам (так называемое термическое запаздывание) обусловлены термическим сопротивлением $R$. Величина $R$ складывается из термосопротивления ячейки калориметра $R_{0}$ и термосопротивления испытываемого образца $R_{s}\left(R=R_{0}+R_{s}\right)$ и определяется по соотношению $R=\operatorname{ctg} \varphi$, где $\varphi-$ угол наклона переднего фронта пика теплоемкости на кривой ДСК.

Термосопротивление ячейки калориметра $R_{0}$ зависит от конструктивных особенностей и определяется для каждого калориметра экспериментальным путем с помощью калибровки эталонами. На рис. 1 (кривая 5) показана эндотерма плавления эталонного индия, помещенного непосредственно в капсулу (в качестве эталона могут использоваться также сплав Вуда и галлий). Из рисунка видно, что линейность переднего фронта эндотермы при увеличении температуры сохраняется и, следовательно, термосопротивление в процессе плавления остается постоянным. Это позволяет определить значение $R_{0}$, 
Коэффициент теплопроводности в нонадекане с добавками и параметры расчета пиков теплоемкости при твердотельном фазовом переходе

\begin{tabular}{|c|c|c|c|c|c|c|c|c|c|}
\hline Образец & $\begin{array}{c}R, \\
\mathrm{~K} \cdot \mathrm{W}^{-1}\end{array}$ & $\begin{array}{c}R_{s}, \\
\mathrm{~K} \cdot \mathrm{W}^{-1}\end{array}$ & $\mathrm{~W} \cdot \stackrel{\lambda,}{\mathrm{m}^{-1} \cdot \mathrm{K}^{-1}}$ & $\begin{array}{c}T_{01}, T_{02}, \\
\mathrm{~K}\end{array}$ & $\begin{array}{c}\Delta C_{\max } \\
\mathrm{J} \cdot \mathrm{g}^{-1} \cdot \mathrm{K}^{-1}\end{array}$ & $\begin{array}{l}q_{1}, q_{2} \\
\mathbf{J} \cdot \mathrm{g}^{-1}\end{array}$ & $\begin{array}{c}q_{n} /\left(q_{1}+q_{2}\right) \\
\%\end{array}$ & B & $\begin{array}{c}\omega, \\
\mathrm{nm}^{3}\end{array}$ \\
\hline \multirow[t]{2}{*}{ I } & \multirow[t]{2}{*}{979} & \multirow[t]{2}{*}{888} & \multirow[t]{2}{*}{$0.1264[5]$} & 291.60 & 18.0 & 35.1 & 77 & 580 & 84 \\
\hline & & & & 292.40 & 11.0 & 10.8 & 23 & 1200 & 570 \\
\hline \multirow[t]{2}{*}{ II } & \multirow[t]{2}{*}{299} & \multirow[t]{2}{*}{208} & \multirow[t]{2}{*}{0.5376} & 292.50 & 15.0 & 17.5 & 44 & 1000 & 290 \\
\hline & & & & 293.15 & 43.0 & 21.9 & 56 & 2300 & 540 \\
\hline \multirow[t]{2}{*}{ III } & \multirow[t]{2}{*}{317} & \multirow[t]{2}{*}{226} & \multirow[t]{2}{*}{0.4928} & 292.50 & 16.0 & 18.7 & 40 & 1000 & 280 \\
\hline & & & & 293.1 & 43.0 & 27.6 & 60 & 2200 & 500 \\
\hline \multirow[t]{2}{*}{ IV } & \multirow[t]{2}{*}{364} & \multirow[t]{2}{*}{273} & \multirow[t]{2}{*}{0.4032} & 291.75 & 10.0 & 11.7 & 38 & 1000 & 430 \\
\hline & & & & 292.30 & 38.0 & 19.3 & 62 & 2300 & 600 \\
\hline
\end{tabular}

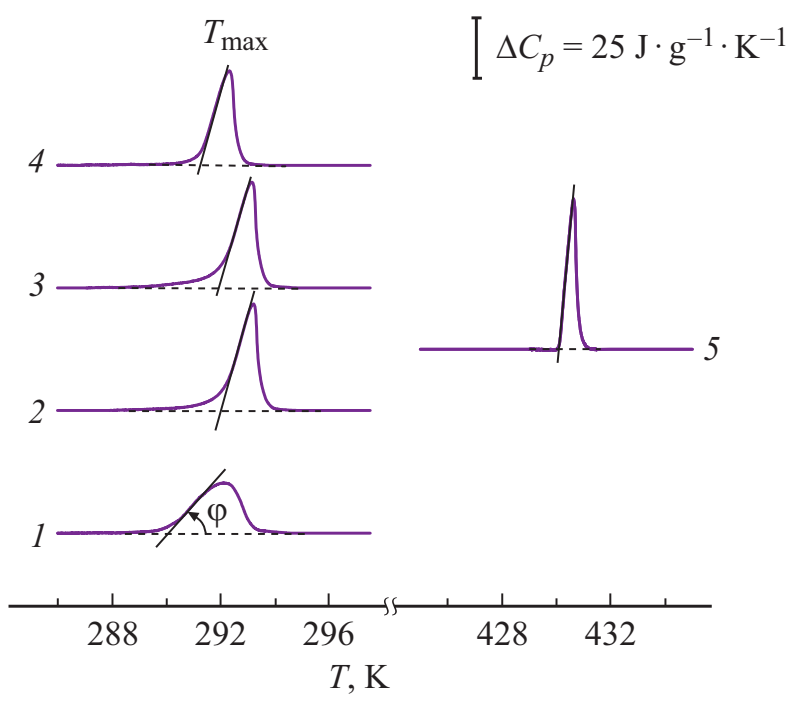

Рис. 1. Эндотермические пики, отвечающие твердофазным переходам в образцах I-IV (кривые $1-4$ соответственно) и эталоне In (кривая 5). Жирные линии - экспериментальные данные, тонкие - линейная экстраполяция переднего фронта пика, штриховые линии - базовые.

величина которого для данного калориметра составила $91 \mathrm{~K} \cdot \mathrm{W}^{-1}$. Таким образом, используя при испытании образцы материала, имеющего ярко выраженную аномалию на зависимости теплоемкости от температуры (например, в виде твердотельного фазового перехода), можно по разнице $R-R_{0}$ определить величину термосопротивления образца $R_{s}$. При этом следует сохранять такие же условия испытания, как при испытании эталона, а именно скорость нагревания, стандартные капсулы и режим газовой продувки калориметрической камеры прибора ДСК.

Значение $R_{s}$ с точностью до множителя $d$, учитывающего геометрические размеры образца, определяет коэффициент теплопроводности материала образца $\lambda$ по соотношению $\lambda=d / R_{s}$. При испытании образцов одинаковой геометрической формы множитель $d$ остается постоянным. Это позволяет находить сравнительные характеристики теплопроводности испытываемых образцов. В том случае, если известен коэффициент теплопроводности одного из образцов, по определенному для него значению $R_{s}$ можно установить значение $d$, которое в дальнейшем можно использовать для определения коэффициента $\lambda$ других образцов.

На рис. 1 изображены кривые ДСК, полученные при нагревании исследуемых образцов в температурном интервале, содержащем диапазон структурного ФП первого рода $(\sim 290-294 \mathrm{~K})$. Из рисунка видно, что форма эндотермических пиков на кривых, соответствующих нонадекану (кривая 1) и композитам (кривые 2-4), существенно различается: у последних значительно возросли амплитуды пиков $\left(\Delta C_{p \max }\right)$ и уменьшились температурные диапазоны фазового превращения. Из рисунка также видно, что линейность переднего фронта эндотерм при увеличении температуры для всех образцов в значительной степени сохраняется и, следовательно, термосопротивление в процессе фазового перехода остается постоянным. По углу наклона переднего фронта $\varphi$ для всех исследуемых образцов были определены значения $R$, которые приведены в таблице.

Как отмечалось выше, для определения коэффициентов $\lambda$ всех образцов достаточно найти значение $d$ одного из испытываемых образцов. Действительно, поскольку коэффициент теплопроводности для парафинов хорошо известен $\left(\lambda=0.1264 \mathrm{~W} \cdot \mathrm{m}^{-1} \cdot \mathrm{K}^{-1}[5]\right)$, нетрудно определить значение множителя, учитывающего геометрические размеры образца, которое для нонадекана оказалось равным $d=112 \mathrm{~m}^{-1}$. Используя это значение, можно определить величину коэффициента теплопроводности для исследуемых композитов. Рассчитанные таким образом значения $\lambda$ представлены в таблице.

Из данных, приведенных в таблице, следует, что теплопроводность (коэффициент теплопроводности $\lambda$ ) исследуемых композитов существенно (в 3-4 раза) превышает теплопроводность большинства аморфнокристаллических полимеров. Следует отметить, что данные, полученные для двух композитов с одинаковыми добавками частиц $\mathrm{Al}_{2} \mathrm{O}_{3}$ размером 50-70 и 90-110 nm, существенно различаются. В первом случае теплопроводность оказалась несколько выше, чем во втором. 


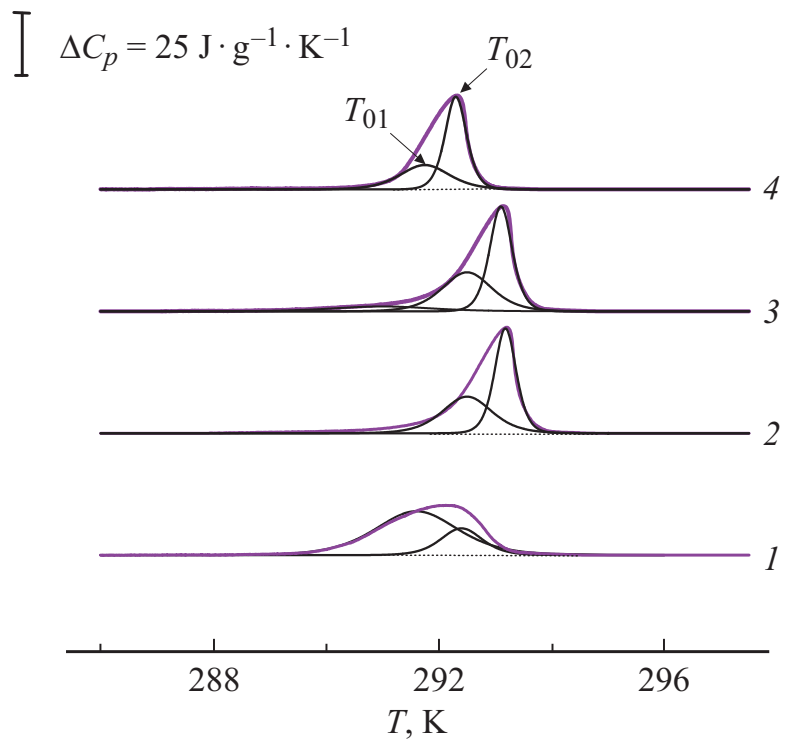

Рис. 2. Эндотермические пики, отвечающие твердофазным переходам первого рода в образцах I-IV (кривые 1-4 соответственно). Жирные линии - экспериментальные данные, тонкие - результат расчета по зависимости (2).

Наибольший эффект от введения наноразмерных частиц наблюдался в исследовании, в котором в парафин было внедрено $\sim 10 \%$ графена [6]. Коэффициент теплопроводности по результатам этой работы составил $\lambda=0.9362 \mathrm{~W} \cdot \mathrm{m}^{-1} \cdot \mathrm{K}^{-1}$. Поскольку теплопроводность твердых тел имеет разную природу в зависимости от типа вещества, можно предположить, что, добавляя в парафин частицы оксида алюминия и серебра, имеющих значительно бо́льшую проводимость (для $\mathrm{Al}_{2} \mathrm{O}_{3} \quad \lambda \approx 25-30 \mathrm{~W} \cdot \mathrm{m}^{-1} \cdot \mathrm{K}^{-1}$, для $\mathrm{Ag}$ $\left.\lambda \sim 400 \mathrm{~W} \cdot \mathrm{m}^{-1} \cdot \mathrm{K}^{-1}\right)$, можно также ожидать увеличения теплопроводности в композите. Термосопротивление как аддитивная величина складывается из термосопротивления парафина и добавки. Принимая последнюю как величину, близкую к нулю, можно ожидать незначительного уменьшения термосопротивления композита и соответственно увеличения теплопроводности. Наши оценки показывают, что эффект от такого повышения не может превосходить $5-10 \%$. Поэтому наблюдаемый нами эффект многократного увеличения коэффициента теплопроводности в композите связан, по-видимому, с изменением структуры парафина при внедрении в него частиц малого размера. Этот факт находит отражение в изменении термодинамических параметров твердотельного фазового перехода.

Из рис. 1 видна несимметричность формы пиков, что может быть обусловлено наличием по крайней мере двух составляющих. Для выявления этих составляющих проводился анализ пиков в рамках термодинамической теории самосогласованного поля [7] применительно к $\Lambda$-образным размытым ФП первого рода. Основная идея теории состоит в том, что в объеме старой фазы происходит локализация многочисленных флуктуаций в ограниченном объеме в виде стабильных зародышей новой фазы - так называемых элементарных объемов фазового превращения $\omega$. В дальнейшем по мере развития перехода движение межфазной границы осуществляется путем последовательных добавлений зародышей с объемом $\omega$ на возникшую межфазную границу. Размеры устойчивых зародышей $\omega$ можно определить исходя из формы пиков $C_{p}(T)$. В работе [8] получено соотношение для температурной зависимости теплоемкости при размытом фазовом переходе в виде

$$
\begin{aligned}
& \Delta C_{p}(T)=4 \Delta C_{\max } \exp \left[B\left(T-T_{0}\right) / T_{0}\right] \\
& \times\left[1+\exp \left[B\left(T-T_{0}\right) / T_{0}\right]\right]^{-2},
\end{aligned}
$$

где $T_{0}$ - температура ФП первого рода, $\Delta C_{\max }-$ максимальное значение теплоемкости при $T=T_{0}, B-$ атермический параметр.

В несимметричных пиках производилось разделение [3] на два пика симметричной $\Lambda$-образной формы при условии равенства энтальпии экспериментально полученного пика $\left(q_{n}\right)$ сумме энтальпий двух симметричных пиков $\left(q_{1}+q_{2}\right)$, т.е. $q_{n}=q_{1}+q_{2}$. При варьировании параметров $T_{0}, \Delta C_{\max }$ и $B$ для каждого из симметричных пиков можно было получить совпадение либо с правым (высокотемпературным с $T_{0}=T_{02}$ ), либо с левым (низкотемпературным с $T_{0}=T_{01}$ ) плечом пика. Наилучшее совпадение расчетных и экспериментальных зависимостей наблюдалось при значениях параметров, указанных в таблице. На рис. 2 показаны результаты расчета $\Lambda$-образных пиков по соотношению (2) и экспериментальные зависимости теплоемкости $\Delta C_{p}(T)$. Параметр $B$ в приведенном выше соотношении (1) coдержит наиболее интересную информацию о физической природе ФП, поскольку он связан с величиной пика теплоемкости $\Delta C_{\max }$

$$
\Delta C_{\max }=q_{0} B / 4 T_{0}
$$

(где $q_{0}-$ теплота превращения) и элементарным объемом превращения $\omega$

$$
B=\omega \rho q_{0} / k T_{0}
$$

(где $k-$ постоянная Больцмана, $\rho-$ плотность).

Параметр $B$ оказывается структурно-чувствительным параметром, поскольку определяет в материалах с размытыми ФП объемы зародышей новой фазы. Из соотношения (2) можно найти значения удельной теплоты (энтальпии) превращения, из соотношения (3) элементарный объем превращения $\omega$ (полагая в первом приближении, что плотность кристаллов парафинов $\left.\rho \sim 0.8 \mathrm{~g} \cdot \mathrm{cm}^{-3}[9]\right)$. Результаты расчета по этим соотношениям приведены в таблице.

Действительно, твердофазный переход в нонадекане происходит в два этапа. Как видно из рис. 2, в исходном нонадекане фазовое превращение в большей части 
кристалла происходит на первой стадии. При этом инициируется зарождение доменов новой фазы размером $\omega=84 \mathrm{~nm}^{3}$ (это следует из соотношения теплот превращения на первой и второй стадиях; см. таблицу). Фазовое превращение приводит к исчерпанию бездефектных областей кристалла и повышению поверхностной энергии вследствие накопления или появления препятствий в оставшейся, т.е. не претерпевшей фазового превращения, части кристалла. На второй стадии фазового перехода в исходном нонадекане фазовое превращение происходит в меньшей (оставшейся) части кристалла с увеличением объема зародышей до $\omega=570 \mathrm{~nm}^{3}$.

Как видно из таблицы, в отличие от исходного нонадекана фазовое превращение в композитах происходит в большей части кристалла в бездефектных областях с большими объемами $\left(\omega=500-600 \mathrm{~nm}^{3}\right)$, что приводит к снижению рассеяния фононов [5] и способствует увеличению теплопроводности кристаллов композитов по сравнению с теплопроводностью исходного нонадекана. В бездефектных областях с большими объемами формируется ламеллярная структура, которая более упорядочена в сравнении с исходной матрицей за счет увеличения ширины ламелей.

Таким образом, установлено, что значительное увеличение теплопроводности обусловлено не ,аддитивной“ добавкой более теплопроводящего материала, а перестройкой надмолекулярной структуры нонадекана вследствие наличия добавочных центров кристаллизации и формирования специфической надмолекулярной структуры н-алкана в композите.

\section{Конфликт интересов}

Авторы заявляют, что у них нет конфликта интересов.

\section{Список литературы}

[1] В.М. Егоров, А.К. Борисов, В.А. Марихин, Письма в ЖТФ, 45 (23), 38 (2019). DOI: 10.21883/PJTF.2019.23.48718.17744 [V.M. Egorov, A.K. Borisov, V.A. Marikhin, Tech. Phys. Lett., 45 (12), 1204 (2019). DOI: 10.1134/S1063785019120058].

[2] A.K. Borisov, V.M. Egorov, V.A. Marikhin, J. Phys.: Conf. Ser., 1236, 012010 (2019). DOI: 10.1088/1742-6596/1236/1/012010

[3] В.М. Егоров, А.К. Борисов, В.А. Марихин, ФТТ, 63 (3), 406 (2021). DOI: 10.21883/FTT.2021.03.50595.225 [V.M. Egorov, A.K. Borisov, V.A. Marikhin, Phys. Solid State, 63 (3), 498 (2021). DOI: 10.1134/S1063783421030069].

[4] V.A. Bershtein, V.M. Egorov, Differential scanning calorimetry of polymers: physics, chemistry, analysis, technology (Ellis Horwood, N.Y., 1994).

[5] Ю.К. Годовский, Теплофизика полимеров (Химия, М., 1982).

[6] M. Li, Appl. Energy, 106, 25 (2013). DOI: 10.1016/j.apenergy.2013.01.031

[7] М. Фишер, Природа критического состояния (Мир, М., 1968).
[8] Г.А. Малыгин, ФТТ, 43 (10), 1911 (2001). [G.A. Malygin, Phys. Solid State, 43 (10), 1989 (2001). DOI: $10.1134 / 1.1410644]$.

[9] D.M. Small, Physical chemistry of lipids (Plenum Press, N.Y.-London, 1986). 